\title{
Der Einzelfall zählt
}

\section{In der Klinischen Sozialarbeit können ethische Grundprinzipien nur allgemeine Leitlinien sein}

\author{
Anton Schlittmaier
}

Prof. Dr. Anton Schlittmaier ist

Hochschullehrer für Grundlagen

Sozialer Arbeit an der Berufsakademie

Sachsen in Breitenbrunn und

Lehrbeauftragter im Masterstudiengang

Klinische Sozialarbeit an der Hochschule Coburg.

E-Mail sca@ba-breitenbrunn.de
Eine Ethik Klinischer Sozialarbeit muss sich vor allem auf Klientenschutz und auf Standards für Ressourcen und Netzwerke gründen. Dabei ist eine enge Verzahnung von Ethik und Empirie bedeutsam: Standards können nur über normative Festlegungen erfolgen; die Auswirkungen von Standards sind jedoch eine empirische Frage.

Klinische Sozialarbeit ist ein in Deutschland junges Projekt. Eine Definition Klinischer Sozialarbeit steht vor der Schwierigkeit, dass sie weder über Arbeitsfelder, Zielgruppen oder Verfahren allein definiert werden kann (Geißler-Piltz/Mühlum/Pauls 2005). Klinische Sozialarbeit findet beispielsweise im psychiatrischen Versorgungssystem, im Krankenhaus, in der Rehabilitation, aber auch in der Kinder- und Jugendhilfe statt. Sie bezieht sich unter anderem auf Kinder und Jugendliche, psychisch Kranke, Abhängigkeitskranke oder chronisch Kranke (ebd.).

Diese einleitenden Hinweise zeigen, dass Klinische Sozialarbeit schwer im Kontext der üblichen Unterscheidungen eingeordnet werden kann. Sie liegt quer $\mathrm{zu}$ diesen. Klinische Sozialarbeit kann in der Suchthilfe, in der Kinder- und Jugendhilfe, im Krankenhaus und in vielen weiteren Handlungsfeldern zur Anwendung kommen.

Neben der Sozialen Arbeit ist das Gesundheitssystem ein weiterer zentraler Referenzpunkt. Das Selbstverständnis der Klinischen Sozialarbeit ist daher einerseits in der Logik Sozialer Arbeit und andererseits in der Logik von Gesundheitsberufen verankert. Hinzu kommen starke Bezüge zur Psychotherapie (Pauls 2004).

Definitionen von Klinischer Sozialarbeit setzen bei der Schwere der zu behandelnden Störung (z. B. Traumata), bei der Intensität der Intervention und der erforderlichen Behandlungskompetenz an (Geißler-Piltz/Mühlum/Pauls 2005). Zielgruppe sind Klienten, deren Problemlagen eine gegenüber der Kompetenz eines grundständig ausgebildeten Sozialarbeiters und Sozialpädagogen hinausgehende Fachlichkeit erfordern. Diese liegt insbesondere im klinischen Bereich, unterscheidet sich von der Kompetenz eines Psychotherapeuten jedoch durch die dezidierte Berücksichtigung der sozialen Umwelt des Klienten.

Eine Ethik Klinischer Sozialarbeit muss die dargelegten Schwierigkeiten reflektieren. Sie kann nicht Ethik eines Arbeitsfeldes (z. B. Suchtkrankenhilfe) oder Ethik in Bezug auf eine Zielgruppe sein. Weiter sind Klärungen in Bezug auf die Zuständigkeit der Medizinethik (Irrgang 1995) sowie einer Ethik der Psychotherapie (Tress/Langenbach 1999) erforderlich.

\section{Vorgaben}

Ein modernes Gesundheits- und Krankheitsverständnis (Homfeldt/Sting 2006) hebt ein bio-psycho-soziales Modell zum Verständnis von Krankheit und Gesundheit hervor. Bezug genommen wird auf Salutogenese und Stärkenorientierung (Geißler-Piltz/Mühlum/Pauls 2005). Hier lässt sich eine Brücke zur Sozialen Arbeit schlagen, die deutlich macht, dass ein modernes Gesundheitswesen und zentrale Traditionen Sozialer Arbeit in eine gemeinsame Richtung weisen: die multifaktorielle Verursachung von Störungen, ihre Einbindung in soziale Zusammenhänge sowie Stärken- und Ressourcenorientierung. Eine starke Anknüpfung der Klinischen Sozialarbeit an die Traditionen Sozialer Arbeit erscheint somit sehr plausibel. Dies schließt eine Berücksichtigung psychotherapeutischer und gesundheitswissenschaftlicher Aspekte nicht aus.

In institutioneller Perspektive sind für Soziale Arbeit die Berufsethiken der Berufsverbände maßgebend. Bezogen auf die Soziale Arbeit in Deutschland sind dies die berufsethischen Prinzipien des Deutschen Berufsverbandes für Soziale 
Arbeit e. V. (DBSH) sowie der International Federation of Social Workers (IFSW). In Bezug auf die Klinische Sozialarbeit wird auf den Code of Ethics der Clinical Social Work Federation (Dentler/Pauls 2000) verwiesen. Damit steht ein Korpus von Prinzipien zur Verfügung, an denen der Praktiker sich orientieren kann (vgl. Kasten unten).

Die Grundprinzipien, welche den Klienten betreffen weisen in dieselbe Richtung. In den berufsethischen Prinzipien des Deutschen Berufsverbandes für Soziale Arbeit wird die politische Dimension stärker akzentuiert (Stichwort: strukturelle Gerechtigkeit). Dies sollte auch für Klinische Sozialarbeit ein zentrales Prinzip sein, insbesondere unter dem Aspekt der Bedeutung des sozialen Kontextes in Bezug auf Gesundheit und Krankheit.

\section{Menschenbilder}

Berufsethische Prinzipien sind durch Berufsverbände gesetzte normative Vor- gaben. Insbesondere aus ethisch-philosophischer Perspektive greift eine ausschließliche Begründung der Berufsethik über Prinzipien, die durch Verbände beschlossen wurden, zu kurz (Schlittmaier 2004). Man wünscht sich eine Verankerung in übergeordneten Menschenbildern und ethischen Theorien (Pieper 1985). Dies basiert nicht nur auf dem Wunsch nach Tiefe und Fundierung, sondern auf dem Bedürfnis, die Prinzipien der Berufsethiken zu interpretieren und im Konfliktfall gegeneinander abwägen zu können.

In einer pluralistischen Gesellschaft wird es nicht möglich sein, für alle Sozialarbeiter und Sozialpädagogen ein bestimmtes Menschbild oder eine ethische Theorie verbindlich zu machen. Neben der Pluralität der Moralen gibt es auch eine Pluralität der Menschbilder und der Ethiken (Nida-Rümelin 1996).

Für die Klinische Sozialarbeit bedeutet dies, dass sie von den maßgeblichen Berufsethiken der Berufsverbände ihren Ausgang nehmen muss. Dies ist bereits

\section{Die Grundprinzipien einer Ethik der Klinischen Sozialarbeit}

werden durch die Berufsethiken der Berufsverbände maßgebend formuliert. Infrage kommen dafür die berufsethischen Prinzipien des Deutschen Berufsverbandes für Soziale Arbeit, der International Federation of Social Workers sowie der Clinical Social Work Federation. Beispielhaft seien genannt:

Aus den berufsethischen Prinzipien des Deutschen Berufsverbands für Soziale Arbeit:

- Katalog der Menschenrechte; Persönlichkeitsrechte; Sozialstaatsgebot

- Würde der Person

- Solidarität und strukturelle Gerechtigkeit

- Integration der Person in die Gesellschaft; Schutz der Person in der Gesellschaft

- Ablehnung von Diskriminierung

- politischer Einsatz für sozial Benachteiligte

- Orientierung an Konsens mit dem Klienten

- Respektierung der Autonomie des Klienten

- Datenschutz

- Wertschätzung und Anerkennung von Kollegen; Beistand und Absprache mit Kollegen

- Loyalität gegenüber dem Arbeitgeber

Aus dem Code of Ethics der Clinical Social Work Federation:

- Würde des Menschen

- Selbstbestimmung des Klienten

- Verbundenheit mit der demokratischen Gesellschaft

- Übernahme von Verantwortung für die geleistete Soziale Arbeit

- Wohlergehen des Klienten

- Konsens, der auf vorhergehender Informiertheit basiert (informed consent)

- Minimierung ungünstiger Wirkungen

- professionelle Grenzen in Bezug auf private Beziehungen zu Klienten

- Datenschutz

- Schutz der Öffentlichkeit eine Entscheidung, da ja als Ausgangspunkt auch die Medizinethik oder die Ethik der Psychotherapie gewählt werden könnte. Auch die Frage, welche Berufsethiken maßgeblich sind, ist Ergebnis eines Diskurses. Die Relevanz, die dem Code of Ethics der Clinical Social Work Federation zugeschrieben wird, ergibt sich aus der Positionierung der Klinischen Sozialarbeit in der Tradition des Clinical Social Work. Gleiches gilt für die Bedeutung der Berufsethiken des Deutschen Berufsverbandes für Soziale Arbeit und der International Federation of Social Workers.

Nur im Lichte von ethischen Theorien können Prinzipien interpretiert und in ihrer Gewichtung bestimmt werden. Klinische Sozialarbeiter müssen deshalb über angemessene ethische Kompetenz verfügen. Diese umfasst erweiterte Grundkenntnisse in ethischer Theorie, insbesondere in Bezug auf zentrale Begriffe wie Menschenwürde, Verantwortung, Wohlergehen, Selbstbestimmung, Konsens und Gerechtigkeit. Auf der Basis dieser Begriffe lassen sich Prinzipien interpretieren, gewichten und auf Einzelfälle beziehen (Schlittmaier 2004).

Klinische Sozialarbeit kommt in der Praxis vielfach vor, ohne dass sie jeweils bereits als solche ausgewiesen ist. Sie liegt in vielfältigen Arbeitsfeldern und in der Arbeit mit differenten Zielgruppen vor. Die Bestimmung einzelner Bereiche der Praxis Sozialer Arbeit als Klinische Sozialarbeit und die damit einhergehende Forderung, diese als Arbeitsfeld für Fachsozialarbeiter mit spezifischer und erweiterter Qualifikation zu reservieren, wird Aufgabe der Zukunft sein. Dies umfasst bestimmende und reflektierende Urteilskraft. Einerseits sind die Teile der Praxis, die Klinische Sozialarbeit darstellen, $\mathrm{zu}$ bestimmen, anderseits ist der Begriff der Klinischen Sozialarbeit in enger Konfrontation mit der Praxis weiterzubilden. Dies umfasst, die Stellung zu den einzelnen Arbeitsfeldern zu klären. Für die Ethik bedeutet dies, das Verhältnis zwischen einer Ethik einzelner Arbeitsfelder (z. B. Kinder- und Jugendhilfe) und einer Ethik Klinischer Sozialarbeit zu reflektieren.

Klinische Sozialarbeit ist somit auf die für maßgeblich befundenen ethischen Prinzipien von Berufsverbänden sowie auf ethische Theorien zu beziehen. Im Rahmen eines oszillierenden Verfahrens 
sind Praxis, berufsethische Prinzipien sowie ethische Theorien wechselweise zu reflektieren (Schlittmaier 2004). Dabei gibt es keinen Algorithmus. Ein strenges Methodenideal ist nicht umsetzbar. Ähnlich wie es in der Hermeneutik kein verall- nen dies nicht gilt. Eine Information über eine Diagnose gegenüber einem Schwerkranken ist nicht immer angemessen. Zwar gilt das Prinzip des informierten Patienten. Jedoch gibt es Fälle und Situationen, in denen ein Recht auf Nicht-wissen-

\section{"Zwar gilt das Prinzip des informierten Patienten; jedoch kann es auch ein Recht des Nicht-wissen- Wollen geben "}

gemeinerungsfähiges, alle Details umfassendes, Vorgehen einer Interpretation gibt (Röd 1994), existiert auch für die Fallbearbeitung kein bis ins Detail feststehendes Schema. Insbesondere ist das Vorverständnis der Praktiker zu reflektieren: Welche in ihrer Lebenswelt verankerten Konzepte eines angemessenen Umgangs mit anderen Menschen - insbesondere Schwächeren - bringen sie mit?

Weiter gibt es für Klinische Sozialarbeit keine vorgegebene Prioritätenliste der Prinzipien (Schlittmaier 2004). Zwar mag meist das Prinzip der Autonomie Vorrang haben, jedoch sind Fälle denkbar, in de-
Wollen besteht (Geißler-Piltz/Mühlum/ Pauls 2005). Die konkrete Entscheidung ist hier immer auf eine eingehende, alle Ebenen berücksichtigende Erörterung bezogen, und kann nicht unter dem Postulat von Prinzipientreue erfolgen (Zoglauer 1998).

\section{Folgerungen}

Das Proprium Klinischer Sozialarbeit ist der Bezug auf schwierige Klienten sowie die Berücksichtigung des sozial-ökologischen Kontextes. Beide Komponenten

\section{Literatur}

DBSH. Internet http://www.dbsh.de/ html/prinzipien.html.

Dentler, P./Pauls, H.: Code of Ethics der Clinical Social Work Federation (Deutsche Übersetzung). Coburg 2000.

Geißler-Piltz/Mühlum, A./Pauls, H.: Klinische Sozialarbeit. München u. a. 2005.

Homfeld, H. G./Sting, S.: Soziale Arbeit und Gesundheit. Eine Einführung. München u. a. 2006.

Irrgang, B.: Grundriss der medizinischen Ethik. München u. a. 1995.

Nida-Rümelin, J.: Theoretische und angewandte Ethik: Paradigmen, Begründungen, Bereiche. In: Nida-Rümelin, J. (Hg.): Angewandte Ethik. Die Bereichsethiken und ihre theoretische Fundierung (2-85). Stuttgart 1996,

Pauls, H.: Klinische Sozialarbeit. Grundlagen und Methoden psycho-sozialer Behandlung. Weinheim 2004.

Pieper, A.: Ethik und Moral. Eine Einführung in die praktische Philosophie. München 1985.

Röd, W.: Der Weg der Philosophie. Band I, II. München 1994.

Schlittmaier, A.: Ethische Grundlagen klinischer Sozialarbeit. Coburg 2004.

Tress, W./Langenbach, M. (Hg.): Ethik in der Psychotherapie. Göttingen 1999.

Zoglauer, Th.: Normenkonflikte - zur Logik und Rationalität ethischen Argumentierens. Stuttgart 1998.

ermöglichen die Abgrenzung zur Sozialen Arbeit. Der Bezug zu schwierigen Klienten (z.B. schwer beeinträchtigte Kinder und Jugendliche; psychisch kranke Menschen; drogen- und alkoholabhängige Menschen, traumatisierte Personen) differenziert von der Sozialen Arbeit. Der Bezug zum sozial-ökologischen Kontext differenziert von der Psychotherapie.

Beide Aspekte sind ethisch relevant. Bei der Zielgruppe handelt es sich um besonders gefährdete, beeinträchtigte Menschen. Dies bedeutet, dass ein besonderer Schutz dieser Klientel erforderlich ist. Dies betrifft insbesondere auch die Grenzen in Bezug auf private und intime Beziehungen. Für den Sozialarbeiter bedeutet es ein herausgehobenes Maß an Verantwortung und Berücksichtigung der Würde des Anderen. Diese Aspekte finden Ausdruck in den Berufsethischen Prinzipien des Code of Ethics der Clinical Social Work Federation.

Die Berücksichtigung des sozial-ökologischen Aspekts rekurriert auf soziale Gerechtigkeit, aber auch auf Mindeststandards in Bezug auf die Erfüllung von Bedürfnissen. Zur Erhaltung von Gesundheit sind Ressourcen erforderlich. Ein Desiderat einer Ethik Klinischer Sozialarbeit wäre, auch hierüber Aussagen zu machen. Dies wird in der gegenwärtigen Diskussion, die den Schutz des Klienten primär fokussiert, teilweise noch vernachlässigt.

\section{Resümee}

Um Soziale Arbeit zu bleiben, muss Klinische Sozialarbeit die Abgrenzung zur Psychotherapie herstellen. Dies geht primär über die Tradition Sozialer Arbeit und ihren Bezug zur sozial-ökologischen Perspektive. In der Ethik gilt es, diesen Aspekt verstärkt zu thematisieren. Hierbei kann auf die bereits existierenden berufsethischen Prinzipien Sozialer Arbeit zurückgegriffen werden. Die Besonderheit einer Ethik Klinischer Sozialarbeit wird primär in der Vertiefung des Klientenschutzes und in einer Spezifizierung der Standards für Ressourcen und Netzwerke liegen. Hier ist eine enge Verzahnung von Ethik und Empirie angesagt: Standards können nur über normative Festlegungen erfolgen; die Auswirkungen von Standards (Ressourcen; Netzwerke) sind jedoch eine empirische Frage. 